Continents manuscrits

Génétique des textes littéraires - Afrique, Caraïbe,

diaspora

$11 \mid 2018$

Écrire le fleuve Congo après Conrad

\title{
Heart of Darkness, extraits du scénario d'Orson Welles
}

\section{Orson Welles}

\section{OpenEdition \\ Journals}

Édition électronique

URL : https://journals.openedition.org/coma/2898

DOI : $10.4000 /$ coma.2898

ISSN : 2275-1742

\section{Éditeur}

Institut des textes \& manuscrits modernes (ITEM)

\section{Référence électronique}

Orson Welles, « Heart of Darkness, extraits du scénario d'Orson Welles », Continents manuscrits [En ligne], 11 | 2018, mis en ligne le 15 octobre 2018, consulté le 13 janvier 2023. URL : http:// journals.openedition.org/coma/2898; DOI : https://doi.org/10.4000/coma.2898

Ce document a été généré automatiquement le 13 janvier 2023.

\section{(c) $(1) \&$}

Creative Commons - Attribution - Pas d'Utilisation Commerciale - Pas de Modification 4.0 International - CC BY-NC-ND 4.0

https://creativecommons.org/licenses/by-nc-nd/4.0/ 


\title{
Heart of Darkness, extraits du scénario d'Orson Welles
}

\author{
Orson Welles
}

\section{Présentation par Jean-Pierre Orban}

1 En juin 1939, Orson Welles (1915-1985) débarque à Hollywood. Il a vingt-quatre ans et il est auréolé du succès retentissant qu'a eu son adaptation radiophonique de La Guerre des mondes (1898) de son presque homonyme, H.G. Wells (1866-1946): la légende raconte que l'émission provoqua des scènes de panique sur la côte est américaine.

2 À Hollywood, George J. Schaefer, le producteur de RKO Radio Pictures, lui offre un contrat d'écriture et de réalisation de films pour une durée initiale de cinq ans. Imprégné d'une culture littéraire étendue qu'il s'est forgée au théâtre et lors d'un long séjour en Europe, en Irlande particulièrement, Welles propose comme premier scénario une adaptation libre du roman de Joseph Conrad, Heart of Darkness. Le scénario témoigne de l'audace artistique du jeune réalisateur: au début du récit, Welles s'adresse lui-même à l'audience et explique le procédé qui sera celui du récit filmé : tout au long, le point de vue (point of vue) sera celui de Marlow, à la manière même dont le roman est écrit. C'est la première fois qu'est utilisée ce qui prendra le nom de " caméra subjective ». Trop grande audace, coûts trop élevés dus à d'autres procédés de mise en scène, difficultés de réalisation avec le matériel lourd de l'époque: les arguments pour refuser le scénario ne manquent pas à G.J. Schaefer ${ }^{1}$. Le scénario de Welles restera à jamais dans ses tiroirs et, comme cela se répétera plus d'une fois, l'artiste américain abandonnera le projet de ce qui aurait dû et pu être son premier long métrage. Un an plus tard, il proposera à la RKO le scénario d'un autre film audacieux mais moins coûteux: ce sera Citizen Kane, qui révolutionnera le langage cinématographique.

3 Le 9 juin 2018, lors de l'après-midi d'études «Écrire le fleuve Congo après Conrad ", Sara Darmayan et Daddy Moanda Kamono ont lu des extraits des dernières pages du scénario d'Orson Welles, adaptées en français et pour la circonstance par Jean-Pierre 
Orban. Les dialogues ont été maintenus dans leur intégralité et les passages du roman de Welles ont été - sauf mention exprès dans les notes - repris de la traduction française du Coeur des ténèbres dans le volume Pléiade 2017 (traduction de Jean Deurbergue $)^{2}$. Les indications de tournage, très détaillées dans le texte original (le mot "caméra " apparaitt plus de six cents fois dans le scénario), ont été réduites aux descriptions d'ambiance.

La scène reproduite ici, traversée par la voix et la silhouette de Kurtz, raconte le retour de Marlow au poste de départ à l'embouchure du fleuve africain, avant de passer à la scène finale sur l'East River à New York. Marlow retrouve Elsa, la fiancée de Kurtz. Originalité du scénario de Welles, la fiancée est nommée et présente en Afrique. Elle a tenté de remonter le fleuve avec Marlow pour retrouver et sauver Kurtz, mais a été forcée de débarquer du bateau en cours de voyage à cause des dangers auxquels elle s'exposait...

Scénario de Welles, avec dessins de scènes sur la page de gauche

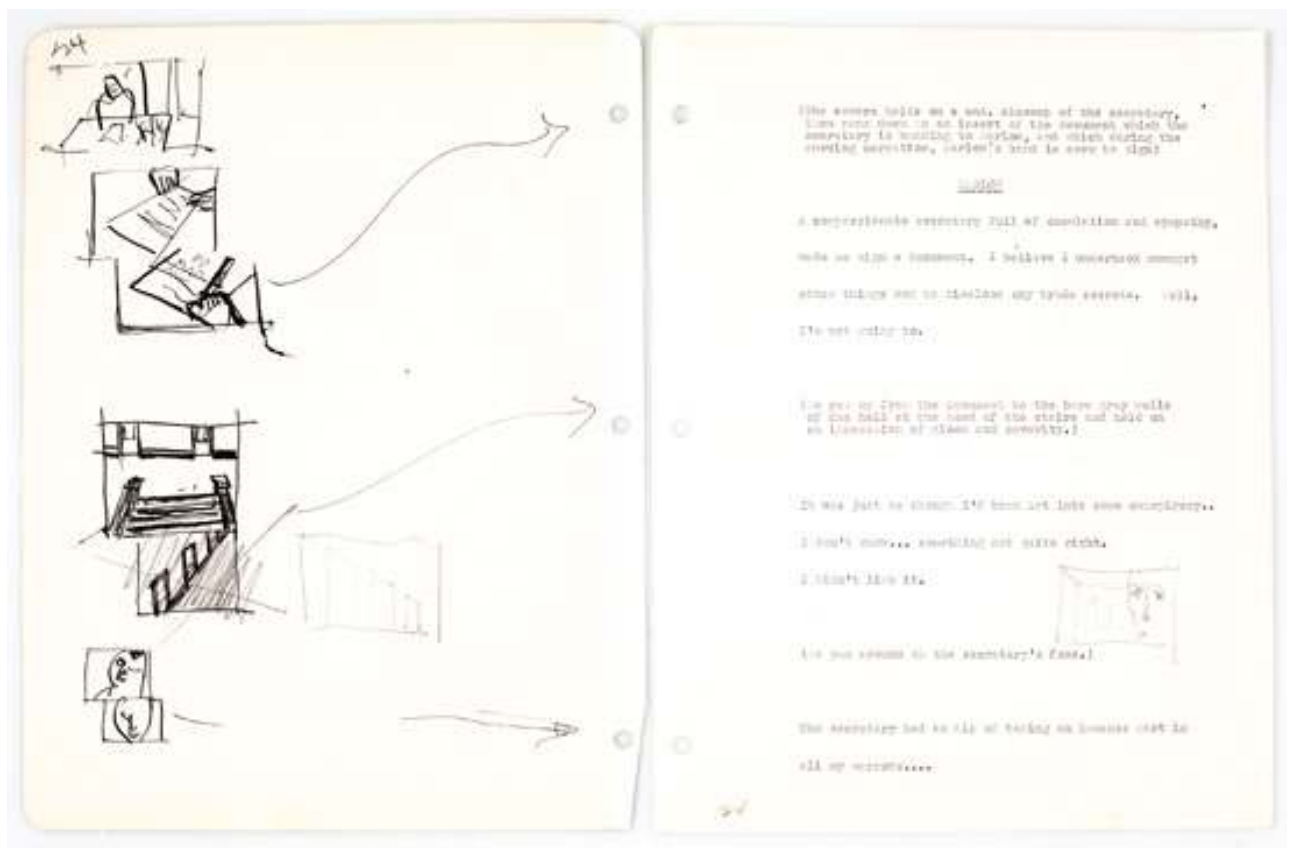

\section{Extérieur bateau (sur le fleuve) et station}

6

7 Je ne veux rien de plus que la justice...

\section{VOIX DE KURTZ}

On entend la voix de Kurtz tout près de nous, mais en même temps étrangement faible.

Une porte, dans le coin au fond de la salle à manger ${ }^{3}$, s'ouvre. Un rayon de lumière blafarde éclaire le mur du fond en tôle ondulée et traverse la silhouette de Kurtz qui y est projetée, effaçant du même coup les dernières images de la jungle et des feux de camp. La tête de Kurtz est encore visible mais s'estompe.

Elsa pénètre (par la porte du fond) et s'arrête, face à la caméra. Tandis qu'elle parle, les accords d'une valse s'insinuent doucement dans la bande son. L'orchestration devrait être ici légère et 
exotique, de manière à ne pas être perçue comme un signal musical, mais comme l'écho d'une vieille mélodie.

$\underline{\text { ELSA }}$

Monsieur Marlow?

VOIX DE KURTZ

Marlow, pourquoi ne prenez-vous pas ma place?

VOIX DE MARLOW (racontant $\left.{ }^{4}\right)$

Je les vis, elle et lui, dans le même moment - sa mort à lui, son chagrin à elle -, je les vis ensemble, je les entendis ensemble.

Pendant cette narration (par la voix de Marlow), Elsa a gratté une allumette et allumé une petite lampe. Elle porte la lampe et se dirige tout en parlant vers la caméra jusqu'au moment où elle se retrouve au centre de l'image, en gros plan. La lumière de la lampe éclaire son visage, atténuant la silhouette de Kurtz. Celle-ci disparaît presque de l'écran à mesure qu'Elsa avance. Seuls subsistent les contours de la silhouette, qui encadrent le visage d'Elsa. L'intensification de la lumière sur le visage d'Elsa est proportionnelle à l'ombre qui s'installe dans le regard de Kurtz.

ELSA (regardant intensément Marlow)

Vous étiez à ses côtés quand il est mort ?

VOIX DE KURTZ

L'horreur ! L'horreur !

À la fin de ces mots, la silhouette de Kurtz et le son de sa voix s'effacent de l'écran, tandis que ses yeux brillent et s'éteignent comme des charbons ardents. Au son de sa voix, meurt le faible murmure de la valse. La voix de Kurtz s'arrête net quand Elsa commence à parler.

ELSA

Vous l'avez bien connu.

VOIX DE MARLOW $^{5}$ (après un court silence)

Je l'ai aussi bien connu qu'il est possible à un homme d'en connaître un autre.

La musique cesse au fur et à mesure que la narration reprend. On entend la voix de Kurtz dans le silence.

\section{VOIX DE KURTZ}

\section{L'horreur!}

$\underline{\text { ELSA }}$

Vous savez quels immenses projets étaient les siens. Il faut que quelque chose demeure. Ses paroles, au moins, ne sont pas mortes.

\section{VOIX DE MARLOW}

Ses paroles demeureront.

$\underline{\text { ELSA }}$

Il est mort comme il a vécu !

VOIX DE MARLOW

Sa fin fut en tout point digne de sa vie.

ELSA

Et je n'étais pas avec lui. 

mère, plus que... lui-même. Il avait besoin de moi ! De moi ! Pardonnez-moi. Je... je... l'ai
pleuré si longtemps en silence - en silence... Vous étiez à ses côtés - jusqu'à la fin?

de la caméra. La musique reprend. La caméra s'éloigne, Elsa apparaît de plus en plus petite, avec tout autour d'elle l'obscurité.

\section{Extérieur des installations, colline et port - nuit - (pluie)}

61 La caméra quitte la pièce en un mouvement arrière, sort par le portail, descend la colline et revient sur le port, en un retournement exact des mouvements au début de l'histoire. Une pluie fine tombe et un voile de brume recouvrent le port. Nous nous éloignons sur l'océan, tandis que la lumière continue à briller dans la salle à manger.

62 Ce mouvement de la caméra ne dure que vingt secondes au plus et s'achève dans le Pays Ténébreux ${ }^{7}$, flou à l'horizon. La musique diminue et Marlow reprend son récit:

63 VOIX DE MARLOW (racontant) 

Mais les cieux ne tombent pas pour une telle vétille. Seraient-ils tombés, je me le demande, si j'avais rendu à Kurtz la justice qui lui était due ?

\section{Extérieur port de New York et bateau de Marlow - nuit}

Plan rapproché [en un mouvement rapide] de Marlow assis dans son bateau. Il craque une allumette pour allumer sa pipe. Marlow regarde la caméra, et à travers elle droit dans les yeux du public. caméra. C'est comme s'il voyait Kurtz au-delà. Il tient toujours son allumette, qui éclaire son visage.

72 MARLOW

73 Je n'ai pas pu. Je n'ai pas pu le lui dire. Cela aurait été trop de noirceur - trop de complète noirceur...

74 (Pause)

75 Nous avons perdu le début du jusant...

76 Marlow lève la tête et jette l'allumette dans l'eau. La caméra suit l'allumette sur le courant. La musique reprend lentement. La caméra se porte sur l'eau vers l'horizon lointain, où brillent les derniers rayons du soleil derrière les nuages sombres.

77 VOIX DE MARLOW (racontant)

78 Le large est barré par un banc de nuages noirs, et la tranquille voie d'eau menant jusqu'aux extrêmes confins de la terre coule, sombre, sous un ciel entièrement couvert... paraît mener jusqu'au coeur d'immenses ténèbres.

79 (FONDU)

80 FIN

\section{NOTES}

1. Voir notamment Jason Bailey, "How Orson Welles Almost Made His Film Debut With an Innovative "Heart of Darkness" Adaptation » : Flavorwire, 6 mai 2015, http://flavorwire.com/ 517572/how-orson-welles-almost-made-his-film-debut-with-an-innovative-heart-of-darknessadaptation ou Louis Mallié, " Heart of Darkness, le plus adacieux projet d'Orson Welles », dans 
Actualitté, 25.08.2014, https://www.actualitte.com/article/culture-arts-lettres/heart-ofdarkness-le-plus-audacieux-projet-d-orson-welles/50849

2. Welles, lui-même, reprend généralement le texte anglais original, en sélectionnant les passages, les redécoupant et les composant en un dialogue ou monologue suivi. Il change parfois, mais rarement, un mot ou le temps d'un verbe (ainsi «I loved him » au lieu de "I love him ») : dans ces cas, la traduction française de la scène a suivi le scénario de Welles.

3. Sans doute un réfectoire.

4. Chaque fois qu'il est écrit « racontant ", Marlow raconte la scène qui se joue dans le scénario (le film).

5. Marlow est hors champ.

6. Ici, en une remarquable intervention, Welles change le temps du verbe « love ».

7. Dark Country.

\section{RÉSUMÉS}

En 1939, Orson Welles (1915-1985) écrit une adaptation cinématographique du Cour des ténèbres de Joseph Conrad. Le film, qui aurait dû être le premier long métrage de l'artiste américain, ne fut jamais réalisé. Voici, adaptées pour une lecture publique, les pages finales du scénario.

In 1939, Orson Welles (1915-1985) wrote a cinematographic adaptation of Joseph Conrad's Heart of Darkness. The movie, which would have been Welles' first feature, was never produced. Here are, adaptated for a public reading, the script's last pages.

\section{AUTEUR}

\section{ORSON WELLES}

ORSON WELLES (1915-1985), réalisateur, scénariste, producteur et acteur américain de cinéma, réalisateur d'émissions radiophoniques et metteur en scène de théâtre. Il est connu, notamment, pour son film Citizen Kane, qui a révolutionné le langage cinématographique.

JEAN-PIERRE ORBAN qui signe l'introduction et l'adaptation des extraits du scénario de Welles, est chercheur associé à l'ITEM. Il a, par ailleurs, traduit et adapté plusieurs oeuvres de l'anglais, dont King Leopold's Soliloquy (satire) de Mark Twain ou Sleep with Me (théâtre) de Hanif Kureishi. 